\title{
Assessment of the Molecular Mechanism of Action of SB3, a Trastuzumab Biosimilar
}

\author{
Kyungyeol Paek ${ }^{1}$ - Go-woon Kim ${ }^{1}$. So Yeong Ahn ${ }^{1}$. Joon Hyuk Lim ${ }^{1}$. Dongkeum Jung ${ }^{1}$ · Seokkyun Kim ${ }^{1}$. \\ Jae Hee Lee ${ }^{1}$ (1)
}

Published online: 23 September 2019

(c) The Author(s) 2019

\begin{abstract}
Background SB3, a biosimilar of Herceptin ${ }^{\circledR}$ (trastuzumab, hereinafter referred to as reference product) is currently approved in the EU, Korea, Australia, the USA, and Brazil for the treatment of human epidermal growth factor receptor (HER) 2-positive early and metastatic breast cancer and HER2-positive metastatic gastric cancer. Previously, the biological similarity of SB3 to EU- or US-sourced reference product was assessed using various cell-based and binding assays.

Objective In this paper, as a part of its similarity assessment, SB3 was evaluated for additional characteristics related to its molecular mechanism of action (MoA).

Methods For extracellular effects of SB3, HER2-overexpressing cancer cell lines were used to assess expression of surface HER2, shedding of the extracellular domain of HER2, and antibody-dependent cell-mediated phagocytosis (ADCP) activity. For intracellular effects, Akt phosphorylation and vascular endothelial growth factor (VEGF) release were assessed. Additionally, in vitro docetaxel or pertuzumab combination experiments were performed for further characterization; anti-proliferation, HER2/HER3 dimerization inhibition, apoptosis, and antibody-dependent cell-mediated cytotoxicity (ADCC) assays were used. Results It was confirmed that SB3 is highly similar to the reference products on quality attributes related to extracellular/ intracellular efficacy. This similarity was also confirmed during combination studies with docetaxel and pertuzumab.

Conclusion Overall, the equivalence of SB3 with reference product in MoA-related qualities in in vitro mono- and combination therapy experiments may support clinical bioequivalence of the two substances.
\end{abstract}

\section{Introduction}

Human epidermal growth factor receptor (HER) 2, which is overexpressed in about $20-25 \%$ of aggressive breast cancer patients, plays a critical role in cell proliferation and anti-apoptotic activity [1]. After HER2 forms a dimer with other members of the HER family, its tyrosine residues are phosphorylated. This phosphorylation leads to the activation of mitogen-activated protein kinase (MAPK) and phosphoinositide-3-kinase-protein kinase B/Akt (PI3 K-PKB/Akt) signal transduction pathways that are upregulated in invasive tumors [2-4]. Numerous studies have demonstrated that HER2-targeting antibody therapies such as trastuzumab

Jae Hee Lee

jaehee81.lee@samsung.com

1 Quality Evaluation Team, Samsung Bioepis Co., Ltd, 107, Cheomdan-daero, Yeonsu-gu, Incheon 21987, Republic of Korea attenuate tumor progression and reduce recurrence rates in patients with HER2-overexpressing cancer [5-7].

Trastuzumab, a monoclonal antibody binding to extracellular subdomain IV of HER2, has been a major therapeutic option in breast cancer in particular, and has demonstrated significant benefit in prolongation of patients' survival [8]. Currently, trastuzumab is being used as the standard of care in HER2-positive early and metastatic breast cancer and HER2-positive metastatic gastric cancer [9, 10]. Trastuzumab's mechanisms of action (MoAs) include the inhibition of proteolytic cleavage of the extracellular domain (ECD) of HER2, reduction of the surface HER2 level on HER2-overexpressing cell lines, and promotion of antibody-dependent cell-mediated phagocytosis (ADCP) by attracting immune cells to tumor cells [11-13]. Moreover, trastuzumab inhibits HER2-mediated cellular signaling, and its treatment results in the inactivation of survival factors such as the PI3 K/Akt pathway and reduction of vascular endothelial growth factor (VEGF) production in the cell [11-13]. More recently, patients administered trastuzumab combination treatments (with pertuzumab and chemotherapeutic agents such as 


\section{Key Points}

SB3, a biosimilar of trastuzumab currently approved in the EU, Korea, Australia, the USA, and Brazil, was characterized in vitro for the purpose of supporting bioequivalence with trastuzumab in terms of its molecular mechanism of action (MoA).

SB3 demonstrated high similarity to the reference product with regards to quality attributes related to extracellular and intracellular mechanisms of action.

Combination studies with docetaxel and pertuzumab confirmed molecular MoA similarity between SB3 and the reference product.

paclitaxel and docetaxel) have shown significant improvement in event-free or overall survival [14, 15]. Multiple studies have demonstrated the effectiveness of trastuzumab combination therapy by demonstrating its anti-proliferative, apoptosis-promotive, and HER2 dimerization-inhibitive activity on HER2-overexpressing breast and gastric cancer cell lines [16, 17].

SB3 is a trastuzumab biosimilar approved by the European Commission (EC) (November 2017), Korea Ministry of Food and Drug Safety (November 2017), Australia Therapeutic Goods Administration (TGA) (December 2018), US Food and Drug Administration (FDA) (January 2019), and Brazilian Health Regulatory Agency (Anvisa) (May 2019).

During its developmental stages, SB3 underwent analytical characterization to confirm its similarity to the reference product $\left(\right.$ Herceptin $\left.^{\circledR}\right)$ in terms of quality. Previous studies on the characterization of SB3 showed the similarity of SB3 to reference product in terms of functional/biological quality attributes as well as clinical efficacy [18, 19]. Herein, as part of a similarity assessment, the additional MoA-related characteristics of SB3 were explored in vitro, both in monoand combination therapy.

\section{Materials and Methods}

\subsection{Materials}

The reference products in this study were selected randomly from 154 lots of EU- and US-sourced reference product. The CellTiter-Blue ${ }^{\circledR}$ and CytoTox-Glo ${ }^{\circledR}$ kits were obtained from Promega (Madison, WI, USA). The phycoerythrin (PE) anti-human CD340 (ErbB2/HER2) antibody was obtained from Biolegend (San Diego, CA, USA). The ErbB2/HER2 enzyme-linked immunosorbent assay (ELISA) kit was obtained from R\&D Systems (Minneapolis, MN, USA), the Phospho-Akt (Ser473) ELISA kit was obtained from Cell Signaling Technology (Danvers, MA, USA), and the Human VEGF Quantikine ${ }^{\circledR}$ ELISA kit was obtained from R\&D Systems. The CellTrace ${ }^{\mathrm{TM}}$ carboxyfluorescein succinimidyl ester (CFSE) Cell Proliferation Kit was obtained from Thermo Fisher Scientific (Waltham, MA, USA), the docetaxel (Taxotere ${ }^{\circledR}$ ) was obtained from Sanofi-Aventis (Paris, France), and the PathHunters ${ }^{\circledR}$ Flash Detection Kit was obtained from DiscoverX (Fremont, CA, USA). The recombinant human heregulin- $\beta 1$ was obtained from Peprotech (Seoul, South Korea) and the Caspase-glo 3/7 assay kit was obtained from Promega.

\subsection{Cell Lines and Cell Culture}

SKBR3 cells were obtained from American Type Culture Collection and cultured at $37{ }^{\circ} \mathrm{C}, 5 \% \mathrm{CO}_{2}$ in McCoy's $5 \mathrm{~A}$ (Gibco, Grand Island, NY, USA) containing 10\% fetal bovine serum (FBS) (Hyclone, Logan, UT, USA). BT-474 cells were obtained from American Type Culture Collection and cultured at $37{ }^{\circ} \mathrm{C}, 5 \% \mathrm{CO}_{2}$ in Dulbecco's Modified Eagle Medium: Nutrient Mixture F-12 (DMEM/F12, Gibco) containing 10\% FBS (Hyclone). NK92-CD16 cells were obtained from Biogen (Cambridge, MA, USA) and cultured at $37{ }^{\circ} \mathrm{C}, 5 \% \mathrm{CO}_{2}$ in Myelocult H5100 (STEMCELL Technology, Vancouver, Canada) containing human interleukin (IL)-2 (Cell Signaling Technology) and puromycin (Gibco). NCI-N87 cells were obtained from American Type Culture Collection and cultured at $37{ }^{\circ} \mathrm{C}, 5 \% \mathrm{CO}_{2}$ in RPMI 1640 (Gibco) containing 5\% FBS (Hyclone). The MDA-MB-175-VII cell line for HER2/HER3 dimerization was obtained from American Type Culture Collection and cultured at $37{ }^{\circ} \mathrm{C}, 5 \% \mathrm{CO}_{2}$ in RPMI 1640 (Gibco) containing $1 \%$ FBS (Hyclone).

\subsection{Surface Human Epidermal Growth Factor Receptor (HER) 2 Expression-Level Measurement}

Measurement of the surface HER2 expression was performed with HER2-overexpressing human breast cancer cell line (SKBR3). $400 \mathrm{ng} / \mathrm{mL}$ of SB3 and reference product were incubated with SKBR3 at $37^{\circ} \mathrm{C}, 5 \% \mathrm{CO}_{2}$ for $24 \mathrm{~h}$. Following incubation, cells were fixed by formalin and labeled with PE-conjugated IgG (R\&D Systems) or PE anti-human CD340 (ErbB2/HER2) antibody (BioLegend). The surface HER2 level was measured by flow cytometry analysis (FACSVerse $^{\mathrm{TM}}$, BD Biosciences, San Jose, CA, USA). This quantified the $\mathrm{PE}$ fluorescence, the subsequent reduction of which showed SB3- or reference product-induced reduction of surface HER2. 


\subsection{HER2 Extracellular Domain (ECD) Shedding Measurement}

To measure HER2 ECD shedding, SB3 and reference product were incubated with a human breast cancer cell line (SKBR3) and 4-aminophenylmercuric acetate (APMA) for $2 \mathrm{~h}$ at $37^{\circ} \mathrm{C}, 5 \% \mathrm{CO}_{2}$. After incubation, the ability of trastuzumab to inhibit HER2 ECD shedding was determined by measuring the soluble HER2 ECD level in growth media using a human ErbB2/HER2 Quantikine ${ }^{\circledR}$ ELISA kit (R\&D Systems).

\subsection{Antibody-Dependent Cell-Mediated Phagocytosis (ADCP)}

Human peripheral blood monocytes (STEMCELL Technologies, Vancouver, Canada) were differentiated into M2c macrophage using RPMI 1640 (Gibco) containing 10\% FBS (Hyclone), $50 \mathrm{ng} / \mathrm{mL}$ of recombinant human macrophage colony-stimulating factor (M-CSF; R\&D Systems), and $50 \mathrm{ng} / \mathrm{mL}$ of recombinant human IL-10 (R\&D Systems). Using a CellTrace ${ }^{\mathrm{TM}}$ CFSE Cell Proliferation Kit, M2c macrophage was stained with fluorescent dye. For comparison, six lots each of SB3 and reference product (final concentration: $69 \mathrm{ng} / \mathrm{mL}$ ) were administered to SKBR3 stained with CellTrace ${ }^{\mathrm{TM}}$ Far Red Cell Proliferation Kit. Following incubation, ADCP was induced for $4 \mathrm{~h}$ and analyzed with FACSVerse $^{\mathrm{TM}}$ (BD Bioscience). ADCP activity was determined by flow cytometry. Target SKBR3 cells were labeled with Far Red dye and monocyte-derived M2c macrophages were labeled with CFSE dye. Phagocytosis was evaluated using a FACSVerse ${ }^{\mathrm{TM}}$ flow cytometer, and the percentage phagocytosis was calculated by dividing the number of doublepositive cells by the total number of macrophage cells.

\subsection{Inhibition of Akt Phosphorylation Measurement}

SB3 and reference product were incubated with a human breast cancer cell line (SKBR3) at $37^{\circ} \mathrm{C}, 5 \% \mathrm{CO}_{2}$ for $1 \mathrm{~h}$. Following incubation, cells were lysed with phosphatase inhibitors (Thermo Fisher Scientific) and the media was harvested. Corresponding ELISA kits (Cell Signaling Technology) were used to determine the levels of total Akt and phosphorylated Akt (Ser473).

\subsection{In Vitro Angiogenesis Assay (Vascular Endothelial Growth Factor [VEGF] Release Assay)}

In vitro angiogenesis regulation was measured using VEGF ELISA to quantify levels of soluble VEGF, which is a known hallmark of angiogenesis. A human breast cancer cell line (BT474) was treated with $0.2 \mu \mathrm{g} / \mathrm{mL}$ of SB3 and reference product and incubated at $37^{\circ} \mathrm{C}, 5 \% \mathrm{CO}_{2}$ for $72 \mathrm{~h}$. Following incubation, spin down was performed at $3500 \mathrm{rpm}$ for 5 min to collect the supernatant. Human VEGF Quantikine ${ }^{\circledR}$ ELISA kit (R\&D Systems) was utilized to detect levels of secreted soluble VEGF.

\subsection{Combination Experiment with Chemotherapy}

An anti-proliferation assay was performed to evaluate the combinational effect of each of SB3 and reference product with chemotherapeutic agents. Docetaxel 0.53-12.5 ng/mL (Taxotere ${ }^{\circledR}$, Sanofi-Aventis) and trastuzumab 24-200 ng/ $\mathrm{mL}$ were prepared with serial dilution and transferred into a BT474-seeded plate. After a 96-h incubation, anti-proliferation activity was measured using a CellTiter-Blue ${ }^{\circledR}$ cell viability assay kit (Promega).

\subsection{Anti-Proliferation Assay}

Anti-proliferation potency was determined using the CellTiter-Blue ${ }^{\circledR}$ cell viability assay kit (Promega) according to the manufacturer's instructions. BT-474 cells were incubated with trastuzumab and/or pertuzumab for 4 days at $37{ }^{\circ} \mathrm{C}$, $5 \% \mathrm{CO}_{2}$ in DMEM/F12 containing 10\% FBS. Anti-proliferation potency was measured using the CellTiter-Blue ${ }^{\circledR}$ cell viability assay kit (Promega) according to the manufacturer's instructions. The relative number of viable cells was quantified by measuring the fluorescence (excitation at $560 \mathrm{~nm}$ and emission at $590 \mathrm{~nm}$ ) using a SpectraMax ${ }^{\circledR}$ M3 (Molecular Devices, Sunnyvale, CA, USA). Data were analyzed using parallel line analysis (PLA, Stegmann Systems $\mathrm{GmbH}$, Rodgau, Germany) software to calculate the relative anti-proliferation potency.

\subsection{HER2/HER3 Dimerization}

For the HER2/HER3 heterodimerization assay, the PathHunter $^{\circledR}$ (DiscoverX) dimerization assay was used to detect ligand-induced dimerization of the two subunits of a receptor-dimer pair. The cells have been engineered to coexpress one receptor subunit fused to Enzyme Donor (ED) and a second dimer partner fused to Enzyme Acceptor (EA). Interaction between the dimer partners forces complementation of the two enzyme fragments, resulting in the formation of a functional enzyme that hydrolyzes a substrate to generate a chemiluminescent signal.

\subsection{Apoptosis}

The luminescence-based assay was used to evaluate apoptotic activity using the Caspase-glo 3/7 assay kit (Promega). The Caspase-glo 3/7 system is a luminescent material that quantifies the active form of caspase- 3 and -7 , which play 
key effector roles in apoptosis in mammalian cells. The density of the luminescent signal, which is proportional to the active form of caspase-3/-7, is measured by the microplate reader (Envision ${ }^{\mathrm{TM}}$, PerkinElmer, Wallac Oy, Finland).

\subsection{Antibody-Dependent Cell-Mediated Cytotoxicity (ADCC) Assay}

The ADCC assay was performed to assess Fc-related biological activity of trastuzumab against a HER2-overexpressing human breast cancer cell line (SKBR3). NK92-CD16 cells, a human natural killer cell line expressing CD16, were used as effector cells. Experimentally, trastuzumab and/or pertuzumab were incubated with SKBR3 and NK92-CD16 cells in flat-bottom 96 -well plates for $4 \mathrm{~h}$ at $37{ }^{\circ} \mathrm{C}, 5 \% \mathrm{CO}_{2}$ in McCoy's 5A media. After incubation, the SKBR3/NK92CD16/sample mixture was incubated with a luminogenic peptide substrate (alanyl-alanylphenylanyl-aminoluciferin; AAF-Glo Substrate) included as part of the CytoTox-Glo ${ }^{\circledR}$ kit. The luminescence signals (dead cell signals) were quantified with an Envision ${ }^{\mathrm{TM}}$ Multilabel Plate Reader (PerkinElmer). Data were analyzed by using PLA software to calculate the relative ADCC activity.

\subsection{Software and Related Instruments}

PLA 2.0/2.1 was obtained from Stegmann Systems GmbH (Rodgau, Germany), the Envision ${ }^{\mathrm{TM}}$ Multilabel Plate Reader was obtained from PerkinElmer, and the SpectraMax ${ }^{\circledR}$ M3 Microplate Reader was obtained from Molecular Devices. FACSVerse ${ }^{\mathrm{TM}}$ was obtained from BD Biosciences. CompuSyn, used for drug combinations and for general dose-effect analysis, was obtained from ComboSyn, Inc. (Paramus, NJ, USA).

\subsection{Statistical Analysis}

Statistical analysis was performed with Minitab ${ }^{\circledR}$ statistic software package (version 17.1.0; Leadtools Technologies, Inc., Charlotte, NC, USA). Comparisons between groups were performed with one-way analysis of variance (ANOVA). A value of $p<0.05$ was considered statistically significant. For the purpose of comparing anti-proliferative activities of SB3 and reference product when each combined with pertuzumab, statistical analysis (two-sample $t$-test) was performed at each concentration between trastuzumab mono-treatment and pertuzumab combination treatment.

\subsection{Synergy Definition for Drug Combination}

Based on the combination index (CI) theorem [20], the effectiveness of the combination is evaluated using the computer software CompuSyn, a drug effect analyzer that allows quantitative determination of drug interactions through a median-effect equation. A CI of $<1,=1$, and $>1$ indicates synergism, additive effect, and antagonism, respectively.

\section{Results}

\subsection{Decrease of Surface HER2 Expression}

The trastuzumab-HER2 interaction results in decreased surface HER2 expression by ubiquitination [21-23]. The degradation of the complex after internalization may ultimately reduce downstream signaling of PI $3 \mathrm{~K}$ and MAPK $[22,24,25]$. To compare the surface HER2 expression level, flow cytometry analysis was performed using PE-conjugated antibodies to the HER2- and HER2-overexpressing cell line (SKBR3) applied with SB3 and EU- and US-sourced reference product. The surface HER2 expression levels decreased significantly with trastuzumab treatment relative to the notreatment control. The PE intensity of the negative control was 19,747 mean fluorescence intensities (MFI) $(p=0.001)$. PE intensities measured as MFI ranged from 13,206 to $15,285 \mathrm{MFI}, 12,924$ to $14,827 \mathrm{MFI}$, and 12,774 to 14,355 MFI for SB3 and EU- and US-sourced reference product, respectively. The results demonstrate that SB3 and reference product have highly similar effects against the surface HER2 expression level in the HER2-overexpressing cell line (Fig. 1a) $(p=0.299)$.

\subsection{Inhibition of HER2 ECD Shedding}

Trastuzumab binds to ECD IV of HER2, the site of proteolytic cleavage [25-28]. When a trastuzumab-HER2 complex forms, the cleavage of the ECD of HER2 is blocked, which prevents the formation of a constantly active membranebound $95 \mathrm{kDa}$ HER2 protein called p95 HER2 [28, 29]. APMA is known as an activator of matrix metalloproteinases in HER2-overexpressing cancer cells, and is used to induce HER2 ECD shedding in in vitro experimental conditions $[28,29]$. To assess the potential to inhibit HER2 ECD shedding, SB3 and EU- and US-sourced reference product were administered to SKBR3 cancer cells in the presence of APMA. Subsequently, the levels of soluble HER2 ECD were measured using a human ErbB2/HER2 quantitative ELISA. The level of HER2 ECD shedding decreased significantly in trastuzumab-treated conditions relative to the no-treatment control. The soluble HER2 levels ranged from 1.39 to $1.92 \mathrm{ng} / \mathrm{mL}, 1.38$ to $2.01 \mathrm{ng} / \mathrm{mL}$, and 1.41 to $1.97 \mathrm{ng} /$ $\mathrm{mL}$ for SB3 and EU- and US-sourced reference product, respectively. The soluble HER2 levels in the no-treatment control ranged from 2.27 to $3.23 \mathrm{ng} / \mathrm{mL}$. The three groups showed no statistically significant difference $(p=0.945)$. The results demonstrate that SB3 and reference product have 
(A)

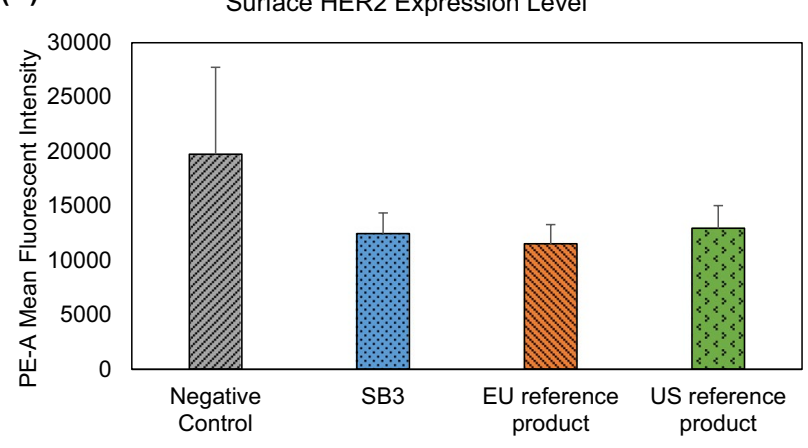

(B)

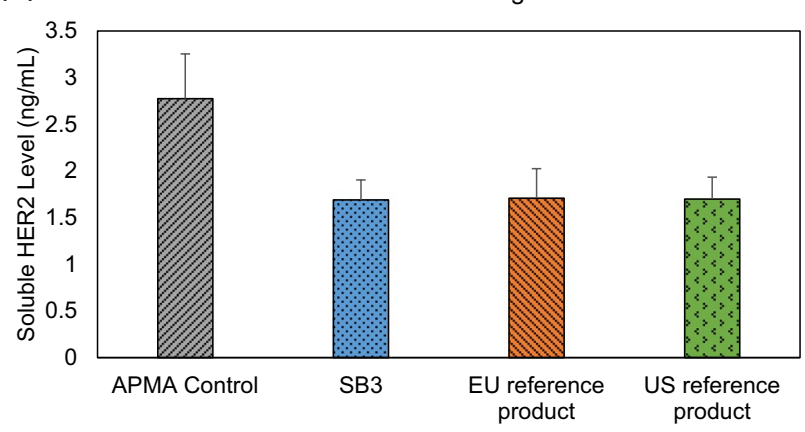

(C)

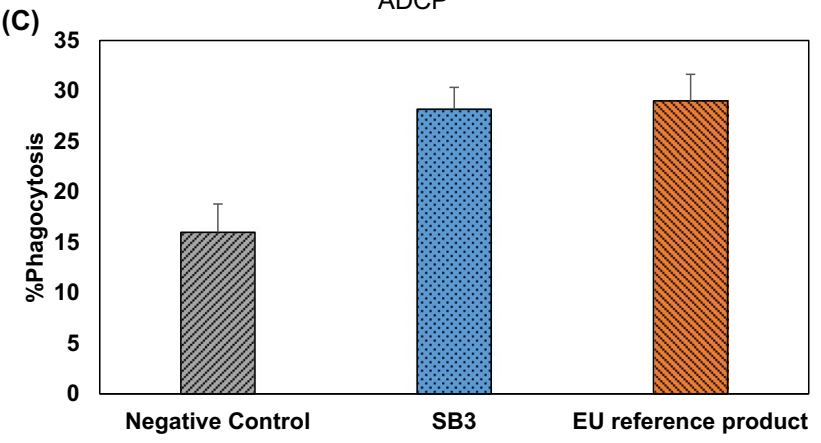

Fig. 1 Comparison of the mechanism of action-related quality attributes of SB3 and reference product (extracellular). a Inhibition of surface HER2 expression level by SB3 $(n=13)$, EU reference product $(n=10)$, and US reference product $(n=7)$ in a breast cancer cell line; b inhibition of HER2 ECD shedding by SB3 $(n=9)$, EU reference product $(n=3)$, and US reference product $(n=6)$ in a breast cancer cell line; and c comparison of ADCP induced by SB3 $(n=6)$ and EU reference product in a breast cancer cell line $(n=6)$. $A D C P$ antibodydependent cell-mediated phagocytosis, $E C D$ extracellular domain, $H E R$ human epidermal growth factor receptor, $P E-A$ phycoerythrinarea

highly similar effects on HER2 ECD shedding on the HER2overexpressing cell line (Fig. 1b).

\subsection{ADCP}

By interacting with $\mathrm{Fc}$ gamma receptor $(\mathrm{Fc} \gamma \mathrm{R})$ on the peripheral blood mononuclear cells (PBMCs) or macrophages, trastuzumab can mediate ADCP against HER2-expressing cancer cells [30, 31]. Increased infiltration of macrophages in the tumor tissue means enhanced efficacy of trastuzumab, whereas depletion of macrophages is equated with decreased anti-tumor activity in vivo [31]. In this sense, the ADCP assay for SB3 and reference product was performed using the SKBR3 cell as a target cell and human monocyte-derived macrophage as an effector cell. There was no significant statistical difference between the percentage phagocytosis of SB3 and that of the EU reference product $(p=0.564)$. The results demonstrate that SB3 and reference product have highly similar potency to induce ADCP on the HER2-overexpressing cell line (Fig. 1c).

\subsection{Inhibition of Akt Phosphorylation}

As HER2 forms a dimer with other members of the HER family, the PI3 K/Akt pathway is activated by phosphorylation of tyrosine residues on the intracellular domain of HER2 $[11,13]$. Several studies have reported that the cytostatic effect of trastuzumab is correlated with inhibition of Akt phosphorylation [24, 25]. To assess the Akt phosphorylation inhibitory activities of SB3 and EU- and US-sourced reference product, phosphorylated Akt (Ser473) levels of SKBR3 cancer cells post-treatment were determined by ELISA. The Akt phosphorylation level was calculated as a relative value against a negative control. Relative levels of phosphorylated Akt (Ser473) ranged from 67\% to $80 \%, 69 \%$ to $78 \%$, and $68 \%$ to $80 \%$ for SB3 and EU- and US-sourced reference product, respectively. The three groups showed no statistically significant difference $(p=0.110)$. The results show that SB3 and reference product have highly similar potency to inhibit Akt phosphorylation on the HER2-overexpressing cell line (Fig. 2a).

\subsection{VEGF Release}

HER2-overexpressing human tumor cells are known to increase angiogenesis, a key role in tumor cell survival and metastasis $[32,33]$. Trastuzumab suppresses angiogenesis by reducing secretion of VEGF, which results in normalization being induced and regression of the vasculature $[32,33]$. Since the decreased level of soluble VEGF reflects down-regulation of angiogenesis [34], the VEGF level was measured after treatment with either SB3 or reference product on the BT474 human breast cancer cell line. In the three groups treated with trastuzumab, VEGF release levels were decreased significantly in comparison with the negative control (322-436 pg/mL). VEGF release levels ranged from 237 to $307 \mathrm{pg} / \mathrm{mL}, 248$ to $286 \mathrm{pg} / \mathrm{mL}$, and 232 to $271 \mathrm{pg} / \mathrm{mL}$ for SB3 and EU- and US-sourced reference product, respectively. The three groups showed no statistically significant difference $(p=0.111)$. The results show 
(A)

Inhibition of Akt Phosphorylation

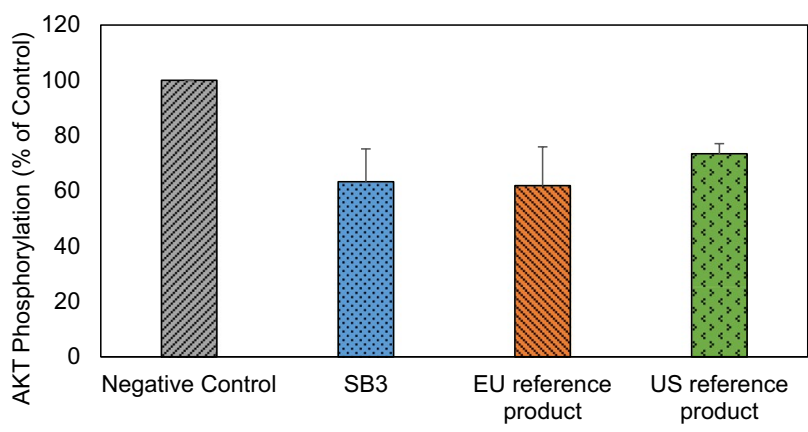

(B)

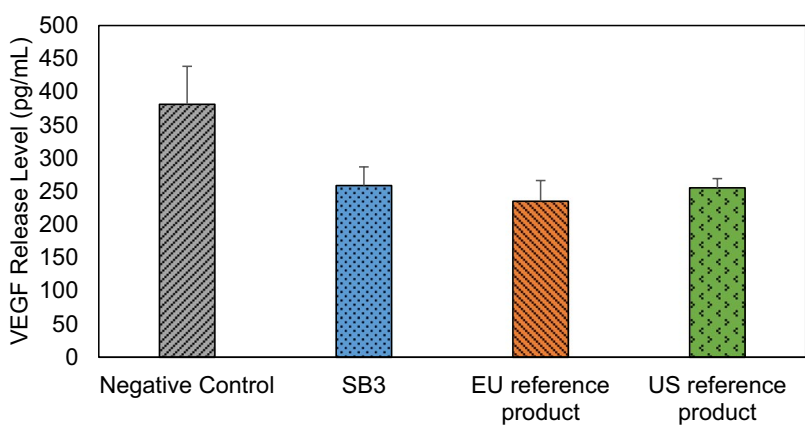

Fig. 2 Comparison of the mechanism of action-related quality attributes of SB3 and trastuzumab (intracellular). a Comparison of Akt phosphorylation reduction level by SB3 $(n=13)$, EU reference product $(n=10)$, and US reference product $(n=7)$ in a breast cancer cell line; data are presented as the relative phosphorylation level of negative control. b Reduction of the VEGF secretion level by SB3 $(n=13)$, EU reference product $(n=10)$, and US reference product $(n=7)$ in a breast cancer cell line. $V E G F$ vascular endothelial growth factor

that SB3 and reference product have highly similar potency to inhibit VEGF secretion in the HER2-overexpressing cell line (Fig. 2b).

\subsection{In Vitro Combination Chemotherapy}

Several clinical trials demonstrated that cytotoxic chemotherapeutic agents work synergistically with trastuzumab when attenuating tumorous activity [34-37]. Since docetaxel has cytotoxic activity on cancer cells by preventing the mitotic cycle, the anti-proliferative effect on the HER2-overexpressing cell line is magnified when used in conjunction with trastuzumab $[35,36]$. To assess the similarity of the in vitro synergistic effect of SB3 and EU- and US-sourced reference product when used with docetaxel, an anti-proliferation assay was performed using a HER2overexpressing breast cancer cell line (BT474). Raw data from the anti-proliferation assay were statistically analyzed using CompuSyn software to calculate a CI, which ranged from 0.50 to $0.96,0.31$ to 0.84 , and 0.50 to 0.98 for SB3 and

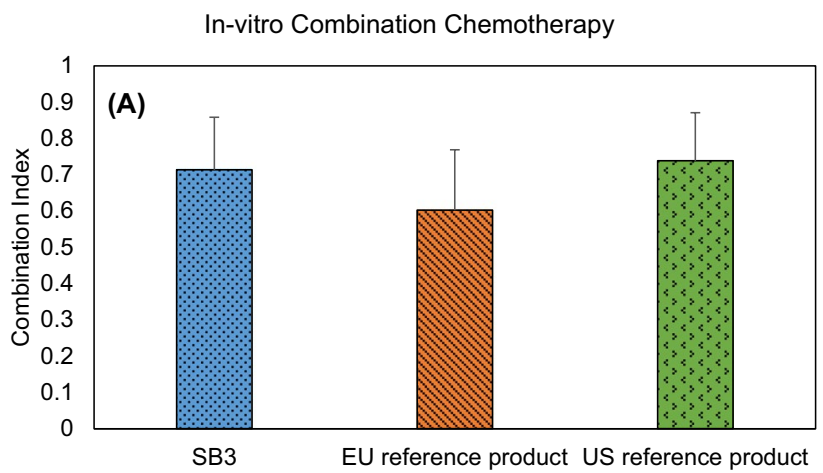

Fig. 3 Comparison of in vitro combination chemotherapy with docetaxel and SB3 or trastuzumab: Akt phosphorylation reduction level by SB3 $(n=6)$, EU reference product $(n=4)$, and US reference product $(n=4)$ in a breast cancer cell line

EU- and US-sourced reference product, respectively. The results show that SB3 and reference product have highly similar potency to induce anti-proliferation on the HER2overexpressing cell line when used in conjunction with cytotoxic chemotherapeutic agents $(p=0.067)$ (Fig. 3).

\subsection{In Vitro Mechanism of Action Assessment in Combination with Pertuzumab}

Pertuzumab targets the dimerization arm (subdomain II) of HER2, which is distinct from the binding site of trastuzumab [38]. As trastuzumab and pertuzumab bind to distinct epitopes on HER2, it has been suggested that a combination of these two agents might provide a more effective inhibitory activity on tumor growth than a mono-treatment [38]. In fact, trastuzumab combination treatment (with pertuzumab and a chemotherapeutic agent such as paclitaxel or docetaxel) has shown significant improvement in event-free or overall survival $[14,15]$. Considering the additive effect of trastuzumab with pertuzumab, MoA-related assays were performed to assess the similarity between SB3 and EUand US-sourced reference product. Two types of cell line (HER2-positive breast and gastric cancer cells, BT474 and NCI-N87) were used to measure anti-proliferation, HER2/ HER3 heterodimerization inhibition, and apoptosis activities of SB3 or reference product when combined with pertuzumab. The synergistic effect was evaluated by calculating the CI using CompuSyn software.

The anti-proliferation assay was performed in both breast and gastric cancer cells. In BT474 (human breast ductal carcinoma), high concentrations $(256-10,000 \mathrm{ng} / \mathrm{mL})$ of both SB3 and reference product showed strong synergistic effects with pertuzumab by showing a meaningful statistical difference in the level of anti-proliferation (Fig. 4a). This pattern was repeated in NCI-N87, a human gastric cancer cell (Fig. 4b). On both human breast ductal and gastric cell lines, 
SB3 and reference product have highly similar synergistic effects on anti-proliferation when used in conjunction with pertuzumab.

The HER2/HER3 dimerization inhibition assay was performed using a PathHunter ${ }^{\circledR}$ Flash Detection Kit with engineered cell line. Trastuzumab does not inhibit HER2/HER3 heterodimerization directly because trastuzumab binds to domain IV, which plays a role in HER2 homodimerization. Nonetheless, due to steric hindrance, an increase in the inhibition of heregulin- $\beta 1$-activated heterodimerization was observed with the addition of trastuzumab to a pertuzumab application. By showing a CI value of 0.59 and 0.63 , respectively, SB3 and the EU-sourced reference product showed highly similar synergistic effects on the inhibition of HER2/HER3 dimerization (dimerization inhibition effect) when used with pertuzumab $(p=0.614)$ (Fig. 5a).

A primary function of HER2 is suppression of apoptosis, thereby increasing cell survival. Several studies demonstrated that trastuzumab/pertuzumab combination induces dramatic tumor regression by inactivating the PI3 K-Akt signaling pathway, which is a main mechanism for inhibiting cell apoptosis [16]. To assess similarity between SB3 and reference product, BT474 and NCI-N87 cell lines were used for the apoptosis assay. Apoptosis activity was measured using the Caspase-glo 3/7 system, which quantifies the active form of caspase- 3 and -7 as key effectors in apoptosis in mammalian cells [39]. When combined with pertuzumab, the caspase-3/-7 activities of reference product and SB3 increased 2.1-fold (standard deviation [SD]: 0.2) and 1.9-fold (SD: 0.2), respectively, in BT474. Likewise, in NCI-N87, caspase-3/-7 activities were increased 1.9-fold (SD: 0.1) and 1.6-fold (SD: 0.3) when treated with reference product and SB3, respectively. In both the BT474 and NCIN87 cell lines, SB3 and the EU reference product showed no significant statistical difference in their abilities to inhibit
Fig. 4 Comparison of in vitro combination treatment with pertuzumab and SB3 or trastuzumab. Anti-proliferation activity by SB3, EU reference product, and combined drug in a BT474 and b NCI-N87. C combination, $P$ pertuzumab, R. $P$ reference product, $T$ trastuzumab, $* p<0.05, * * p<0.01$, $* * * p<0.001$
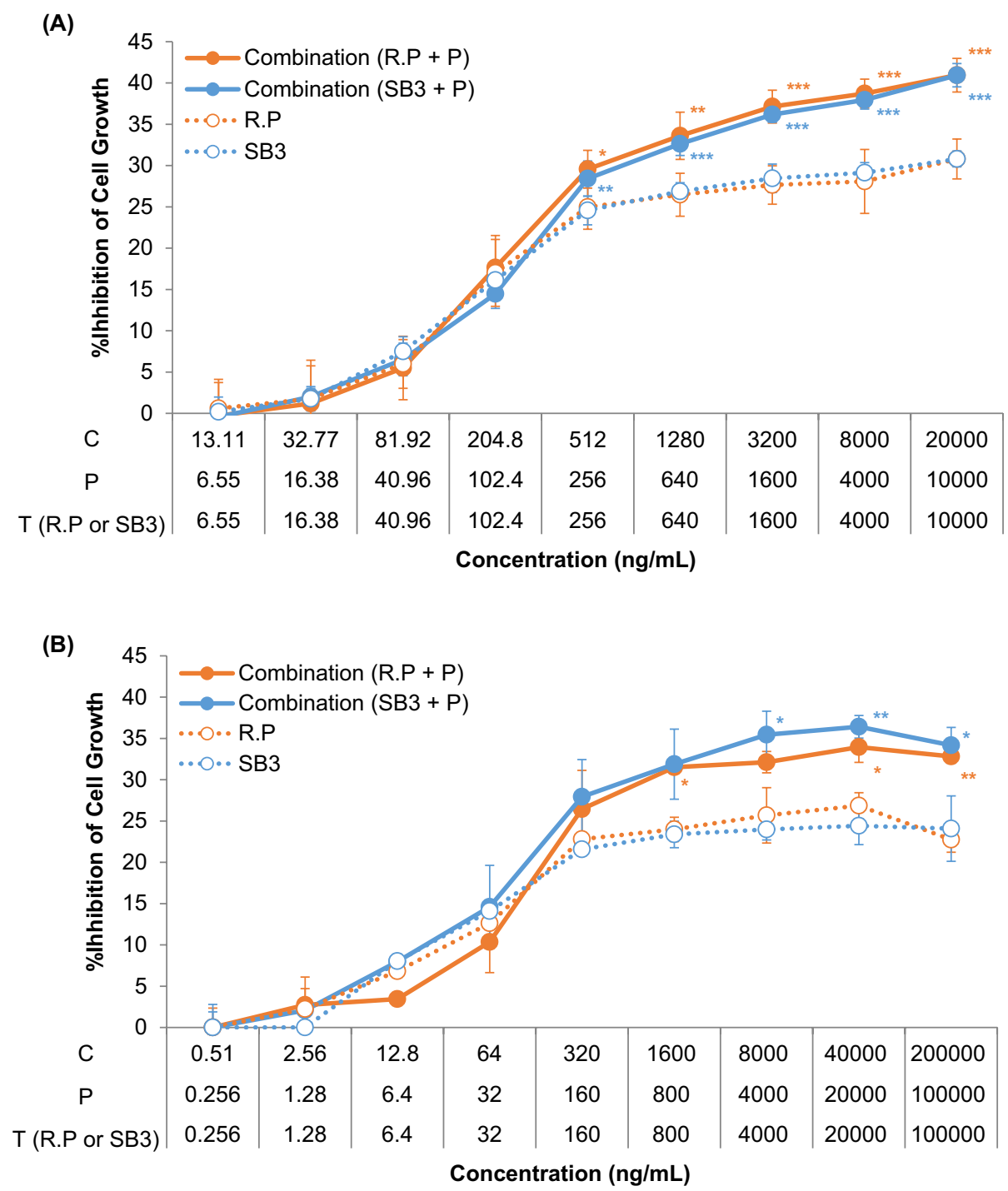
(A)

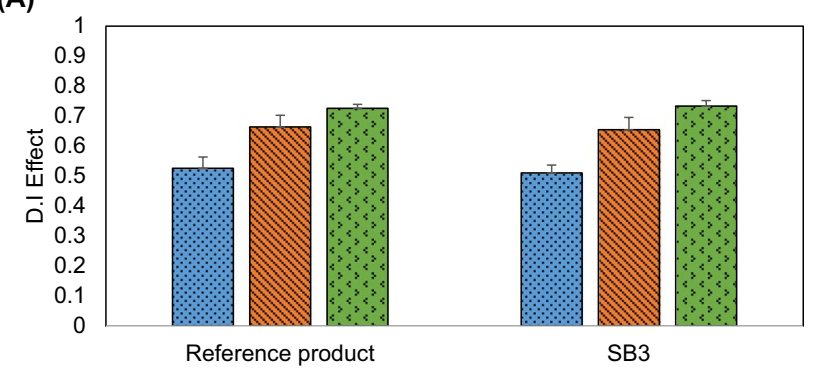

- Trastuzumab (SB3 or reference product) $₫$ Pertuzumab 回 Combination

(B)

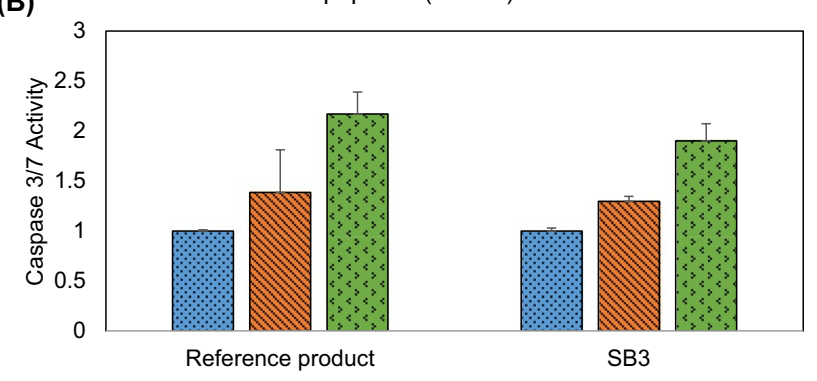

๑Trastuzumab (SB3 or reference product) 소 Pertuzumab 0 Combination

$$
\text { (C) }
$$

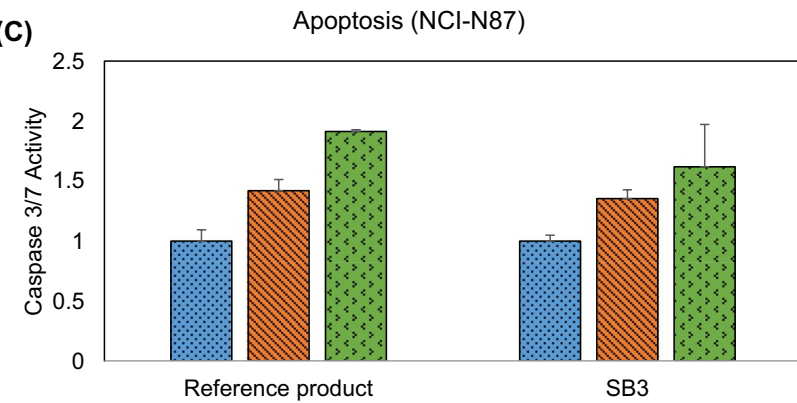

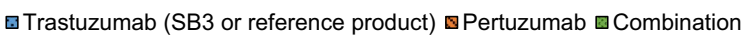

(D)

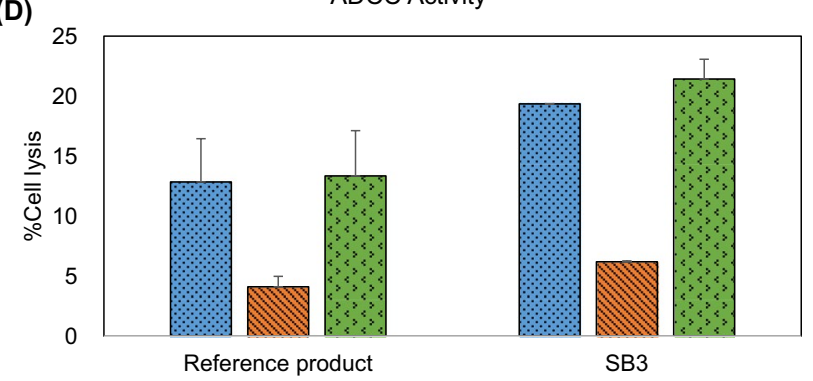

๑Trastuzumab (SB3 or reference product) $\$$ Pertuzumab 0 Combination

Fig. 5 Comparison of the in vitro combination treatment with pertuzumab and SB3 or trastuzumab. Combination effect of SB3 and reference product on a HER2/HER3 heterodimerization inhibition, b apoptosis in BT474, $\mathbf{c}$ apoptosis in NCI-N87, and $\mathbf{d}$ ADCC activity in in breast cancer cell line. ADCC antibody-dependent cell-mediated cytotoxicity, D.I dimerization inhibition, HER human epidermal growth factor receptor anti-apoptotic effects when each was used in conjunction with pertuzumab ( $p=0.286$ and 0.193 , respectively).

As many studies have demonstrated, the anti-tumor efficacy of trastuzumab as a result of antibody-dependent cell-mediated cytotoxicity (ADCC) is enhanced when combined with pertuzumab [17, 40, 41]. To assess similarity between SB3 and reference product, ADCC was performed using a Cytotox-Glo ${ }^{\mathrm{TM}}$ kit with SKBR3 and NK92-CD16. ADCC activities in the linear dose range of the drug concentration were calculated using percentage cell lysis (\%cell lysis). Although a statistical synergistic effect $(\mathrm{CI}<0.9)$ was not observed, the \%cell lysis of SB3 and reference product showed similar effects with respects to ADCC when combined with pertuzumab ( $p=0.077$ ) (Fig. 5d).

\section{Discussion}

The biological characteristics of SB3 based on the critical quality attribute (CQA) classification showed that SB3 exhibits highly similar functional activities to reference product in terms of both CQAs and non-CQAs [19]. Equivalence in clinical efficacy between SB3 and reference product was demonstrated using the ratio of breast pathologic complete response (bpCR) rates in HER2-positive early breast cancer [42]. In this report, analytical similarity assessment was performed in terms of the MoA of trastuzumab in vitro, alone or in combination.

To support the bioequivalence in clinical efficacy demonstrated previously in the phase III study of SB3 [43], additional MoA-related characterizations were performed on trastuzumab: surface HER2-level expression (Fig. 6a), HER2 ECD shedding activity (Fig. 6b), and ADCP activity (Fig. 6e). Results from this study demonstrated that both SB3 and reference product effectively inhibit surface HER2 expression, increase HER2 ECD shedding activity, and elevate ADCP activity. As reported earlier, the phenotype with aggressive HER2 overexpression on the cancer cell and high soluble HER2 levels in the patient's serum was correlated with poor prognosis and increased metastasis [43, 44]. Equivalence in in vivo efficacy can be indirectly seen by proving the equivalence between SB3 and reference product that blocks the increased HER2 signal in in vitro assay platforms.

Regarding the intracellular repercussions of trastuzumab, Akt phosphorylation levels (Fig. 6c) and secreted VEGF levels (Fig. 6d) were compared post-treatment with SB3 and reference product. When SB3 or reference product is administered to a HER2-overexpressing cell line, the intracellular signaling cascade for tumorigenesis is restrained by down-regulation of phosphorylation of Akt, among others [24, 25]. Diminished intracellular pro-survival signaling promotes cell cycle arrest and apoptosis, which may also lead 


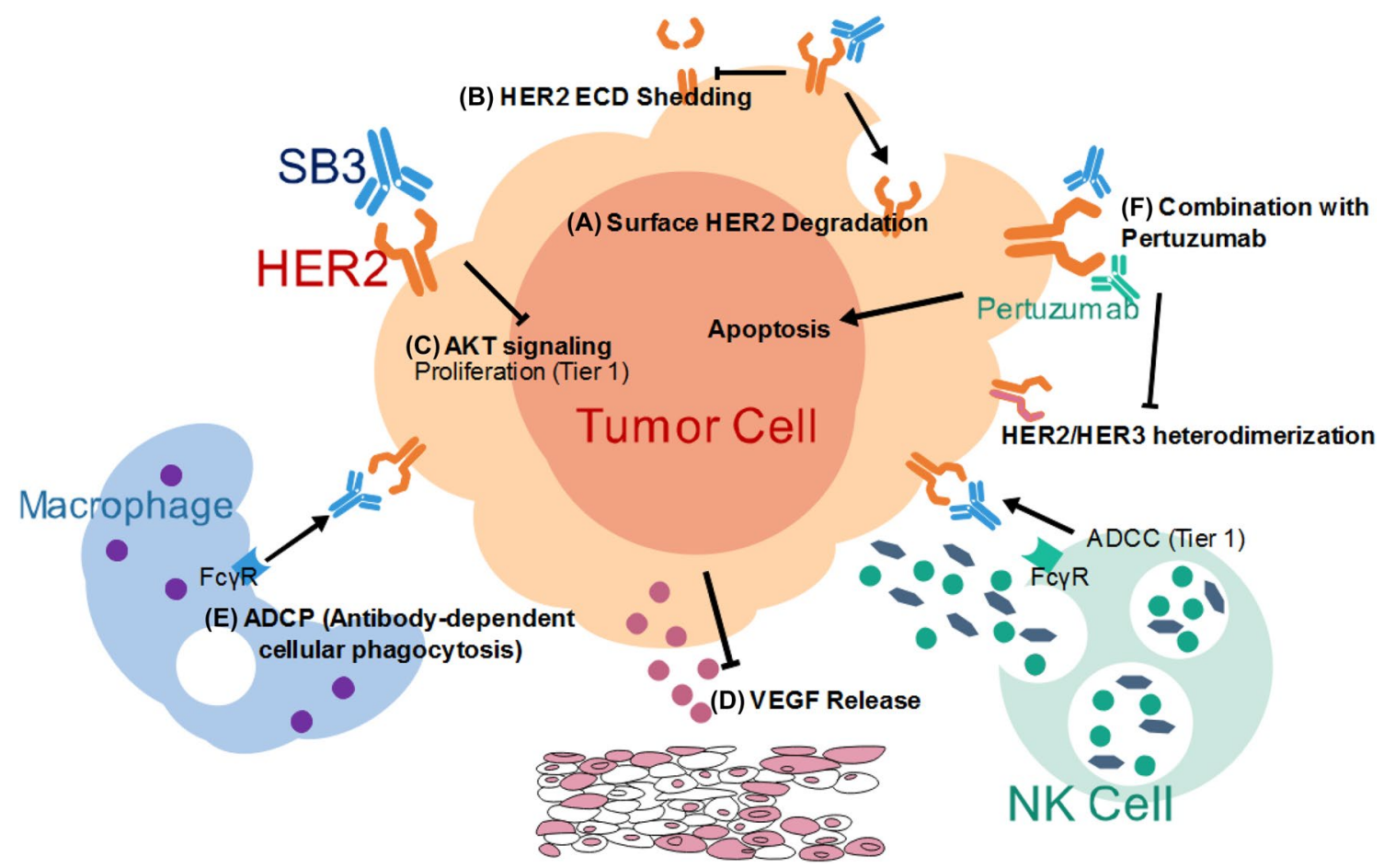

Fig. 6 Overview of the mechanism of action of SB3 on in vitro mono- and combination therapy. A Regulation of surface HER2 expression level, $\mathbf{B}$ inhibition of HER2 ECD shedding, $\mathbf{C}$ downregulation of Akt signaling cascade, $\mathbf{D}$ inhibition of VEGF secretion, $\mathbf{E}$ $\mathrm{ADCP}$, and $\mathbf{F}$ synergistic anti-tumor activity in combination with per-

to reduced tumor volume [24, 25]. In this study, levels of phosphorylated Akt (Ser473) were comparable in SB3- and reference product-treated HER2-overexpressing cells, which resulted in similar anti-proliferation activities.

In clinical anti-tumor therapy, trastuzumab may be used as a standalone therapy. However, to take advantage of potential synergistic effects with other chemotherapeutic agents, trastuzumab has recently been used in tandem with these other treatments [35-37]. As reported in numerous studies, combination of trastuzumab and pertuzumab increases the rate of overall survival among patients with aggressive HER2-positive breast cancer [16, 17]. Therefore, as part of a full and comprehensive similarity assessment of trastuzumab, evaluation based on in vitro combination was performed. Our results demonstrated that SB3 and reference product have a similar synergistic effect when used with docetaxel or pertuzumab (Fig. 6f). In particular, both SB3 and reference product are highly similar with regard to preventing proliferation in in vitro pertuzumab combination experiments. In addition, each group showed CI values less than 0.1. Furthermore, inhibition of the HER2/HER3 dimerization and induction of apoptosis in in vitro combinations of trastuzumab (SB3 and reference product) and pertuzumab were analyzed and a synergistic effect was established for both trastuzumab groups tested; in combination testing of tuzumab (Perjeta ${ }^{\circledR}$ ). ADCC antibody-dependent cell-mediated cytotoxicity, $A D C P$ antibody-dependent cell-mediated phagocytosis, $E C D$ extracellular domain, $H E R$ human epidermal growth factor receptor, $N K$ natural killer, $V E G F$ vascular endothelial growth factor

both dimerization inhibition and apoptosis assays, both SB3 and reference product showed CI values below 0.8 .

In an in vitro ADCC combination assay performed on two separate cell lines, BT474 and NCI-N87, synergistic effects were not observed. Since trastuzumab and pertuzumab bind to domain IV and domain II of HER2, respectively, a synergistic effect could be expected in in vivo combination therapy $[38,46]$. However, recent studies have shown that in vitro combination treatment had no additive effect [41, 47]. The discrepancy between in vivo and in vitro studies can be explained by the fact that an antibody-mediated killing effect of NK cells may be saturated under experimental conditions [47]. This lack of synergistic effect was observed in both SB3 and the reference product.

\section{Conclusion}

In this study, additional characterization of SB3 on the MoAs was performed using the HER2-overexpressing cell line, and showed similar activities to the reference product. Synergistic effects of SB3 with other therapeutic agents were also found to be equivalent to those of the reference product in combination experiments. Therefore, it is concluded that SB3 and reference product have highly similar MoA-related 
qualities in in vitro mono- and combination therapy experiments, which show high correlations with the clinical efficacies of the respective materials.

Acknowledgements We would like to thank Kyung Ah Kim for internal review and proofreading of the manuscript.

\section{Compliance with Ethical Standards}

Funding This work was funded by Samsung Bioepis Co., Ltd.

Conflict of interest Kyungyeol Paek, Go-woon Kim, So Yeong Ahn, Joon Hyuk Lim, Dongkeum Jung, Seokkyun Kim, and Jae Hee Lee are employees of Samsung Bioepis. All authors declare that they have no conflicts of interest that might be relevant to the contents of this manuscript.

Disclaimer The combination studies described herein were performed solely to observe the effect of the presence of pertuzumab on the main modes of action of biosimilar trastuzumab at an in vitro cell-based level. The views expressed in this article are those of the authors alone and are made in a scientific capacity only. It should not be inferred that the authors, or Samsung Bioepis, are making any legal or regulatory representations regarding trastuzumab or pertuzumab. Nothing in this article should be taken to promote, encourage, or endorse use of biosimilar trastuzumab outside the scope of any authorized therapeutic indications or inside the scope of any valid intellectual property rights.

Open Access This article is distributed under the terms of the Creative Commons Attribution-NonCommercial 4.0 International License (http://creativecommons.org/licenses/by-nc/4.0/), which permits any noncommercial use, distribution, and reproduction in any medium, provided you give appropriate credit to the original author(s) and the source, provide a link to the Creative Commons license, and indicate if changes were made.

\section{References}

1. Nahta R, Yu D, Hung MC, Hortobagyi GN, Esteva FJ. Mechanisms of disease: understanding resistance to HER2-targeted therapy in human breast cancer. Nat Clin Pract Oncol. 2006;3(5):269_ 80. https://doi.org/10.1038/ncponc0509.

2. Nida I, Naveed I. Human epidermal growth factor receptor 2 (HER2) in cancers: overexpression and therapeutic implications. Mol Biol Int. 2014;2014:852748. https://doi. org/10.1155/2014/852748.

3. Tarik R, Targeting RTK. Signaling pathways in cancer. Cancers (Basel). 2015;7(3):1758-84. https://doi.org/10.3390/cancers703 0860.

4. Ping W, Zhixiang W. Epidermal growth factor receptor cell proliferation signaling pathways. Cancers (Basel). 2017;9(5):52. https ://doi.org/10.3390/cancers9050052.

5. Del Mastro L, Lambertini M, Bighin C, Levaggi A, D’Alonzo A, Giraudi S, et al. Trastuzumab as first-line therapy in HER2positive metastatic breast cancer patients. Expert Rev Anticancer Ther. 2012;12(11):1391-405. https://doi.org/10.1586/era.12.107.

6. Lantz E, Cunningham I, Higa GM. Targeting HER2 in breast cancer: overview of long-term experience. Int J Womens Health. 2009;1:155-71.
7. Damodaran S, Olson EM. Targeting the human epidermal growth factor receptor 2 pathway in breast cancer. Hosp Pract. 2012;40(4):7-15.

8. Bartsch R, Rottenfusser A, Wenzel C, Dieckmann K, Pluschnig $\mathrm{U}$, Altorjai G, et al. Trastuzumab prolongs overall survival in patients with brain metastases from Her2 positive breast cancer. J Neurooncol. 2007;85(3):311-7. https://doi.org/10.1007/s1 106 0-007-9420-5.

9. Eva N, Alberto Z, Matteo F, Marta R, Martina B, Giovanni C, et al. Effectiveness of trastuzumab in first-line HER2+ metastatic breast cancer after failure in adjuvant setting: a controlled cohort study. Oncologist. 2014;19(12):1209-15. https://doi.org/10.1634/ theoncologist.2014-0227.

10. Goebel SU, Iwamoto M, Raffeld M, Gibril F, Hou W, Serrano J, et al. Her-2/neu expression and gene amplification in gastrinomas: correlations with tumor biology, growth, and aggressiveness. Cancer Res. 2002;62(13):3702-10.

11. Thuy V, Francois XC. Trastuzumab: updated mechanisms of action and resistance in breast cancer. Front Oncol. 2012;2:62. https://doi.org/10.3389/fonc.2012.00062.

12. Gajria D, Chandarlapaty S. HER2-amplified breast cancer: mechanisms of trastuzumab resistance and novel targeted therapies. Expert Rev Anticancer Ther. 2011;11(2):263-75.

13. Nahta R, Esteva FJ. Herceptin: mechanisms of action and resistance. Cancer Lett. 2006;232(2):123-38.

14. Labidi S, Mejri N, Lagha A, Daoud N, El Benna H, Afrit M, et al. Targeted therapies in HER2-overexpressing metastatic breast cancer. Breast Care (Basel). 2016;11(6):418-22. https:// doi.org/10.1159/000452194.

15. Swain SM, Kim SB, Cortés J, Ro J, Semiglazov V, Campone M, et al. Pertuzumab, trastuzumab, and docetaxel for HER2-positive metastatic breast cancer (CLEOPATRA study): overall survival results from a randomised, double-blind, placebo-controlled, phase 3 study. Lancet Oncol. 2013;14(6):461-71. https://doi. org/10.1016/S1470-2045(13)70130-X.

16. Nahta R, Hung MC, Esteva FJ. The HER-2-targeting antibodies trastuzumab and pertuzumab synergistically inhibit the survival of breast cancer cells. Cancer Res. 2004;64(7):2343-6.

17. Yamashita-Kashima Y, Iijima S, Yorozu K, Furugaki K, Kurasawa M, Ohta M, et al. Pertuzumab in combination with trastuzumab shows significantly enhanced antitumor activity in HER2positive human gastric cancer xenograft models. Clin Cancer Res. 2011;17(15):5060-70. https://doi.org/10.1158/1078-0432. CCR-10-2927.

18. Lamb YN. SB3 (Ontruzant ${ }^{\circledR}$ ): a trastuzumab biosimilar. BioDrugs. 2018;32(3):293-6. https://doi.org/10.1007/s40259-018-0282-5.

19. Lee JH, Paek K, Moon JH, Ham S, Song J, Kim S. Biological characterization of SB3, a trastuzumab biosimilar, and the influence of changes in reference product characteristics on the similarity assessment. BioDrugs. 2019;33(4):411-22. https://doi. org/10.1007/s40259-019-00362-5.

20. Chou TC, Talalay P. Quantitative analysis of dose-effect relationships: the combined effects of multiple drugs or enzyme inhibitors. Adv Enzyme Regul. 1984;22:27-55.

21. Klapper LN, Waterman H, Sela M, Yarden Y. Tumor-inhibitory antibodies to HER-2/ErbB-2 may act by recruiting $\mathrm{c}-\mathrm{Cbl}$ and enhancing ubiquitination of HER-2. Cancer Res. 2000;60(13):3384-8.

22. Cuello M, Ettenberg SA, Clark AS, Keane MM, Posner RH, Nau MM, et al. Down-regulation of the erbB-2 receptor by trastuzumab (herceptin) enhances tumor necrosis factor-related apoptosis-inducing ligand-mediated apoptosis in breast and ovarian cancer cell lines that overexpress erbB-2. Cancer Res. 2001;61(12):4892-900. 
23. Austin CD, De Mazière AM, Pisacane PI, van Dijk SM, Eigenbrot C, Sliwkowski MX, et al. Endocytosis and sorting of ErbB2 and the site of action of cancer therapeutics trastuzumab and geldanamycin. Mol Biol Cell. 2004;15(12):5268-82.

24. Junttila TT, Akita RW, Parsons K, Fields C, Lewis Phillips GD, Friedman LS, et al. Ligand-independent HER2/HER3/PI3K complex is disrupted by trastuzumab and is effectively inhibited by the PI3 K inhibitor GDC-0941. Cancer Cell. 2009;15(5):429-40. https ://doi.org/10.1016/j.ccr.2009.03.020.

25. Nagata Y, Lan KH, Zhou X, Tan M, Esteva FJ, Sahin AA, et al. PTEN activation contributes to tumor inhibition by trastuzumab, and loss of PTEN predicts trastuzumab resistance in patients. Cancer Cell. 2004;6(2):117-27.

26. Ménard S, Pupa SM, Campiglio M, Tagliabue E. Biologic and therapeutic role of HER2 in cancer. Oncogene. 2003;22(42):6570-8.

27. Baselga J, Albanell J, Molina MA, Arribas J. Mechanism of action of trastuzumab and scientific update. Semin Oncol. 2001;28(5 Suppl 16):4-11.

28. Molina MA, Codony-Servat J, Albanell J, Rojo F, Arribas J, Baselga J. Trastuzumab (herceptin), a humanized anti-Her2 receptor monoclonal antibody, inhibits basal and activated Her2 ectodomain cleavage in breast cancer cells. Cancer Res. 2001;61(12):4744-9.

29. Tsé C, Gauchez AS, Jacot W, Lamy PJ. HER2 shedding and serum HER2 extracellular domain: biology and clinical utility in breast cancer. Cancer Treat Rev. 2012;38(2):133-42. https://doi. org/10.1016/j.ctrv.2011.03.008.

30. Petricevic B, Laengle J, Singer J, Sachet M, Fazekas J, Steger G, et al. Trastuzumab mediates antibody-dependent cell-mediated cytotoxicity and phagocytosis to the same extent in both adjuvant and metastatic HER2/neu breast cancer patients. J Transl Med. 2013;12(11):307. https://doi.org/10.1186/1479-5876-11-307.

31. Shi Y, Fan X, Deng H, Brezski RJ, Rycyzyn M, Jordan RE, et al. Trastuzumab triggers phagocytic killing of high HER2 cancer cells in vitro and in vivo by interaction with $\mathrm{Fc} \gamma$ receptors on macrophages. J Immunol. 2015;194(9):4379-86. https://doi. org/10.4049/jimmunol.1402891.

32. Kumar R, Yarmand-Bagheri R. The role of HER2 in angiogenesis. Semin Oncol. 2001;28(5 Suppl 16):27-32.

33. Ciesielski M, Szajewski M, Pęksa R, Lewandowska MA, Zieliński J, Walczak J, et al. The relationship between HER2 overexpression and angiogenesis in gastric cancer. Medicine (Baltimore). 2018;97(42):e12854. https://doi.org/10.1097/MD.0000000000 012854.

34. Moreira IS, Fernandes PA, Ramos MJ. Vascular endothelial growth factor (VEGF) inhibition-a critical review. Anticancer Agents Med Chem. 2007;7(2):223-45.

35. Burris HA. Docetaxel (Taxotere) plus trastuzumab (Herceptin) in breast cancer. Semin Oncol. 2001;28(1 Suppl 3):38-44.

36. Pienta KJ. Preclinical mechanisms of action of docetaxel and docetaxel combinations in prostate cancer. Semin Oncol. 2001;28(4 Suppl 15):3-7.
37. Romond EH, Perez EA, Bryant J, Suman VJ, Geyer CE Jr, Davidson NE, et al. Trastuzumab plus adjuvant chemotherapy for operable HER2-positive breast cancer. N Engl J Med. 2005;353(16):1673-84.

38. Franklin MC, Carey KD, Vajdos FF, Leahy DJ, de Vos AM, Sliwkowski MX. Insights into ErbB signaling from the structure of the ErbB2-pertuzumab complex. Cancer Cell. 2004;5(4):317-28.

39. Shin S, Sung BJ, Cho YS, Kim HJ, Ha NC, Hwang JI, et al. An anti-apoptotic protein human survivin is a direct inhibitor of caspase-3 and -7. Biochemistry. 2001;40(4):1117-23.

40. Scheuer W, Friess T, Burtscher H, Bossenmaier B, Endl J, Hasmann M. Strongly enhanced antitumor activity of trastuzumab and pertuzumab combination treatment on HER2-positive human xenograft tumor models. Cancer Res. 2009;69(24):9330-6. https ://doi.org/10.1158/0008-5472.CAN-08-4597.

41. El-Sahwi K, Bellone S, Cocco E, Cargnelutti M, Casagrande F, Bellone $\mathrm{M}$, et al. In vitro activity of pertuzumab in combination with trastuzumab in uterine serous papillary adenocarcinoma. $\mathrm{Br} \mathbf{J}$ Cancer. 2010;102(1):134-43. https://doi.org/10.1038/sj.bjc.66054 48.

42. Pivot X, Bondarenko I, Nowecki Z, Dvorkin M, Trishkina E, Ahn $\mathrm{JH}$, et al. Phase III, randomized, double-blind study comparing the efficacy, safety, and immunogenicity of SB3 (trastuzumab biosimilar) and reference trastuzumab in patients treated with neoadjuvant therapy for human epidermal growth factor receptor 2-positive early breast cancer. J Clin Oncol. 2018;36(10):968-74. https://doi.org/10.1200/JCO.2017.74.0126.

43. Souder C, Leitzel K, Ali SM, Demers L, Evans DB, Chaudri-Ross HA, et al. Serum epidermal growth factor receptor/HER-2 predicts poor survival in patients with metastatic breast cancer. Cancer. 2006;107(10):2337-45.

44. Jensen BV, Johansen JS, Price PA. High levels of serum HER-2/ neu and YKL-40 independently reflect aggressiveness of metastatic breast cancer. Clin Cancer Res. 2003;9(12):4423-34.

45. Hatjiharissi E, Xu L, Santos DD, Hunter ZR, Ciccarelli BT, Verselis $\mathrm{S}$, et al. Increased natural killer cell expression of CD16, augmented binding and ADCC activity to rituximab among individuals expressing the FcyRIIIa-158 V/V and V/F polymorphism. Blood. 2007;110(7):2561-4.

46. Cho HS, Mason K, Ramyar KX, Stanley AM, Gabelli SB, Denney DW Jr, et al. Structure of the extracellular region of HER2 alone and in complex with the Herceptin Fab. Nature. 2003;421(6924):756-60.

47. Tóth G, Szöőr Á, Simon L, Yarden Y, Szöllősi J, Vereb G. The combination of trastuzumab and pertuzumab administered at approved doses may delay development of trastuzumab resistance by additively enhancing antibody-dependent cell-mediated cytotoxicity. MAbs. 2016;8(7):1361-70. 